\title{
$q$-Floor Diagrams computing Refined Severi Degrees for Plane Curves
}

\author{
Florian Block $1 \|^{t}$ \\ ${ }^{1}$ Mathematics Institute, University of Warwick, United Kingdom
}

\begin{abstract}
The Severi degree is the degree of the Severi variety parametrizing plane curves of degree $d$ with $\delta$ nodes. Recently, Göttsche and Shende gave two refinements of Severi degrees, polynomials in a variable $q$, which are conjecturally equal, for large $d$. At $q=1$, one of the refinements, the relative Severi degree, specializes to the (non-relative) Severi degree.

We give a combinatorial description of the refined Severi degrees, in terms of a $q$-analog count of Brugallé and Mikhalkin's floor diagrams. Our description implies that, for fixed $\delta$, the refined Severi degrees are polynomials in $d$ and $q$, for large $d$. As a consequence, we show that, for $\delta \leq 4$ and all $d$, both refinements of Göttsche and Shende agree and equal our $q$-count of floor diagrams.

Résumé. Le degré de Severi est le degré de la variété de Severi paramétrisant les courbes planes de degré $d$ à $\delta$ noeuds. Récemment, Göttsche et Shende ont donné deux raffinements des degrés de Severi, polynomiaux en la variable $q$, qui sont conjecturalement égaux pour $d$ assez grand. Pour $q=1$, un des ces raffinements, le degré de Severi relatif, se spécialise en le degré de Severi (non relatif).

Nous donnons une description combinatoire des degrés de Severi raffinés, en fonction d'un comptage $q$-analogue des "floor diagrams" de Brugallé et Mikhalkin. Notre decription implique que, pour $\delta$ fixé, les degrés de Severi raffinés sont polynomiaux en $d$ et $q$, pour $d$ grand. On montre que, par conséquent, pour $\delta \leq 4$ et pour tout $d$, les deux raffinements de Göttsche et Shende coïncident et sont égaux à notre $q$-analogue de "floor diagrams".
\end{abstract}

Keywords: Severi degree, refined Severi degree, Göttsche conjecture, floor diagram, node polynomial, q-analog

\section{Introduction}

A $\delta$-nodal curve is a reduced (not necessarily irreducible) curve with $\delta$ simple nodes and no other singularities. The Severi degree $N^{d, \delta}$ is the degree of the Severi variety parametrizing plane $\delta$-nodal curves of degree $d$. Equivalently, $N^{d, \delta}$ is the number of $\delta$-nodal plane curves of degree $d$ through $\frac{(d+3) d}{2}-\delta$ generic points in the complex projective plane $\mathbb{C P}^{2}$.

Severi degrees are generally difficult to compute. Their study goes back to the midst of 19th century, when Steiner [Ste48], in 1848, showed that the degree $N^{d, 1}$ of the discriminant of $\mathbb{C P}^{2}$ is $3(d-1)^{2}$. Only in 1998, Caporaso and Harris [CH98] computed $N^{d, \delta}$ for any $d$ and $\delta$, by their celebrated recursion (involving relative Severi degrees $N^{d, \delta}(\alpha, \beta)$ counting curves satisfying tangency conditions to a fixed line).

Di Francesco and Itzykson [DFI95], in 1994, conjectured the numbers $N^{d, \delta}$ to be polynomial in $d$, for fixed $\delta$ and $d$ large enough. In 2009, Fomin-Mikhalkin [FM10] showed that, for each $\delta \geq 1$, there is a polynomial $N_{\delta}(d)$ in $d$ with $N^{d, \delta}=N_{\delta}(d)$, provided that $d \geq 2 \delta$. The polynomials $N_{\delta}(d)$ are called node polynomials.

In 1997, Göttsche [Göt98] made his famous conjecture about universal polynomiality of Severi degrees of arbitrary smooth projective surfaces $S$ with a sufficiently ample line bundle $\mathcal{L}$. Specifically, Göttsche conjectured, for each fixed $\delta$, the existence of one polynomial that, evaluated at appropriate Chern classes of

\footnotetext{
${ }^{\dagger}$ Partially supported by the EPSRC grant EP/I008071/1. 
$S$ and $\mathcal{L}$, equals the Severi degree of $(S, \mathcal{L})$. The Göttsche conjecture was proved by Tzeng [Tze10] in 2010. A second proof was given shortly afterwards by Kool, Shende, and Thomas [KST11]. In the latter proof, the authors identified the numbers $N^{d, \delta}$ (and, in fact, the Severi degrees of any smooth projective surface) as coefficients of the generating function of the topological Euler characteristic of relative Hilbert schemes (see Section 2).

Inspired by this description, Göttsche and Shende [GS11] hypothesized the existence of refined invariants $\tilde{N}^{d, \delta}(q)$. These polynomials in $q$ are defined as the coefficients of a similar generating function, but with the topological Euler characteristic replaced by (a specialization of) the Hodge polynomial. By specializing at $q=1$, one recovers the Severi degree $N^{d, \delta}$ for $d \geq \delta$. More generally, Göttsche and Shende defined refined invariants for any smooth projective surface.

The refined invariants $N^{d, \delta}(q)$ have many nice properties. In particular, for $d$ large enough, they should satisfy a refined Caporaso-Harris recursion (see Formula 2.2), which, at $q=1$, specializes to classical formula of Caporaso and Harris [CH98]. Via this refined recursion, one can define polynomials $N^{d, \delta}(q)$, which Göttsche and Shende call refined Severi degrees. Göttsche and Shende verified that $\tilde{N}^{d, \delta}(q)=$ $N^{d, \delta}(q)$, for $\delta \leq 10$ for $\frac{\delta}{2}+1 \leq d \leq 14$, and conjecture equality for all $\delta \geq 0$ and $d \geq \frac{\delta}{2}+1$ (c.f. Conjecture 2.3 .

In this paper, we study the refined Severi degrees $N^{d, \delta}(q)$. We give a purely combinatorial, positive formula for $N^{d, \delta}(q)$ (Theorem 3.8. This formula is in terms of a $q$-statistic on a class of decorated graphs called floor diagrams (see Section 3). Our new statistic is a $q$-analog of the one of Brugallé and Mikhalkin [BM09] who gave a combinatorial formula for the Severi degrees $N^{d, \delta}$. Theorem 3.8 is a $q$-analog of their [BM09, Theorem 3.6] for the refined Severi degrees $N^{d, \delta}(q)$. In particular, our combinatorial formula 3.1 gives an answer, for $d \geq \delta$ and modulo the conjectured equality $N^{d, \delta}(q)=\tilde{N}^{d, \delta}(q)$ for large $d$, to a question of Göttsche and Shende: "What do $\tilde{N}^{d, \delta}(q)$ count?"

It follows from the definition of the refined invariants $\tilde{N}^{d, \delta}(q)$ that they satisfy universal polynomiality: for each $\delta$, there is a polynomial $\tilde{N}_{\delta}(d ; q)$ in $d$ and $q$ such that $\tilde{N}^{d, \delta}(q)=\tilde{N}_{\delta}(d ; q)$, provided that $d \geq \delta$. Using our combinatorial description, we show a similar result for $N^{d, \delta}(q)$.

Theorem 1.1 For fixed $\delta \geq 1$, there is a (combinatorially defined) polynomial $N_{\delta}(d ; q)$ in $d$ and $q$, such that

$$
N_{\delta}(d ; q)=N^{d, \delta}(q)
$$

provided that $d \geq \delta$.

We call the $N_{\delta}(d ; q)$ refined node polynomials. Together with Göttsche and Shende's observation [GS11] that $\tilde{N}^{d, \delta}(q)$ and $N^{d, \delta}(q)$ agree for $d \leq 14$ and $\delta \leq 10$, we obtain the following.

Corollary 1.2 For $\delta \leq 4$ and any $d \geq \frac{\delta}{2}+1$, we have $\tilde{N}^{d, \delta}(q)=N^{d, \delta}(q)$.

Corollary 1.3 For $\delta \leq 4$ and any $d \geq \frac{\delta}{2}+1, \tilde{N}^{d, \delta}(q)$, as a polynomial in $q$, has non-negative integral coefficients.

Our combinatorial description of the polynomials $N^{d, \delta}(q)$ allows for effective computation of the refined node polynomials; for details see Remark 4.5

Proposition 1.4 For $\delta \leq 2$ resp. $\delta \leq 7$, the polynomials $N_{\delta}(d ; q)$ are as listed in Remark 4.5 resp. on the author's webpage [Blolla].

A natural question is whether the $q$-analog weight on floor diagrams lifts to a $q$-weight on tropical plane curves. In the forthcoming and joint paper [BG12] with Göttsche, we show that this is indeed the case.

Göttsche and Shende also observed a connection between refined invariants and real algebraic geometry. Specifically, they conjectured that $\tilde{N}^{d, \delta}(-1)$ equals $(-1)^{\delta}$ times the tropical Welschinger invariant $W_{\text {trop }}^{d, \delta}$ (for the definition and details see [IKS09]), for $d \geq \frac{\delta}{3}+1$, and confirmed it for $\delta \leq 10$ and $d \leq 14$. Furthermore, by definition, the refined Severi degree $N^{d, \delta}(q)$ specializes, at $q=-1$ and for all $d$, to $(-1)^{\delta} W_{\text {trop }}^{d, \delta}$, as the refined recursion, at $q=-1$, becomes Itenberg, Kharlamov, and Shustin's recursion for $W_{\text {trop }}^{d, \delta}\left[\right.$ IKS09, Theorem 3]. The numbers $W_{\text {trop }}^{d, \delta}$, in turn, equal counts of real plane curves (i.e., complex plane curves invariant under complex conjugation), counted with a sign, through particular configurations of 
real points [Shu05, Proposition 6.1]. Indeed, at $q=-1$, the new $q$-statistic on floor diagrams specializes to the "real multiplicity" of Brugallé and Mikhalkin [BM09], and Theorem 3.8 becomes [BM09, Theorem 3.9] for the numbers $N^{d, \delta}(-1)=(-1)^{\delta} W_{\text {trop }}^{d, \delta}$.

We expect our methods to compute refined Severi degrees also for other toric surfaces. Specifically, we expect the argument to generalize to toric surfaces of " $h$-transverse" polygons, along the lines of [AB10] (see Remark 4.6. Notice that such surfaces are in general not smooth and are thus outside the realm of the (non-refined) Göttsche conjecture [Göt98].

A main contribution of this paper is that we show how the computation of the Severi degrees $N^{d, \delta}$ by Brugallé and Mikhalkin's floor diagrams [BM09] can be refined to compute refined Severi degrees. The proof that the proposed $q$-weights satisfy the refined recursion of Göttsche and Shende (Formula 2.2) is rather straightforward. The existence of such weights on floor diagrams, however, is the possibly surprising novelty.

This paper is organized as follows. In Section 2, we define, following Göttsche and Shende, the refined invariants $\tilde{N}^{d, \delta}(q)$ and the refined Severi degrees $N^{d, \delta}(q)$. In Section 3 we discuss our $q$-analog combinatorial count of floor diagrams, which lets us compute refined Severi degrees. Finally, in Section 4 , we discuss how this description implies Theorem 1.1. Complete proofs of all results will appear in a forthcoming paper [BG12].

\section{Refined Severi Degrees}

In this section, mostly based on [GS11], we review Göttsche and Shende's definition of the closely related notions of the refined invariants and the refined Severi degrees, in the special case of $\mathbb{C P}^{2}$. The definitions are quite technical, however we give an explicit and combinatorial (conjecturally equivalent) description in Section 3, which we then use to prove our results. Recall that the Severi degree $N^{d, \delta}$ is the degree of the Severi variety parametrizing $\delta$-nodal plane curves of degree $d$. Equivalently, $N^{d, \delta}$ is the number of such curves through $\frac{(d+3) d}{2}-\delta$ generic points in $\mathbb{C P}^{2}$.

Plane curves of degree $d$ are parametrized by $\mathbb{C P}\left(\begin{array}{c}\left({ }^{+2}\right)-1 \\ 2\end{array}=H^{0}\left(\mathbb{C P}^{2}, \mathcal{O}_{\mathbb{C P}^{2}}(d)\right)\right.$, the homogeneous degree$d$ polynomials in 3 indeterminants. For $\delta \geq 0$, let $\mathbb{C P}^{\delta}$ be a $\delta$-dimensional subspace of $H^{0}\left(\mathbb{C P}^{2}, \mathcal{O}_{\mathbb{C P}^{2}}(d)\right)$. Let $\mathcal{C} \rightarrow \mathbb{C P}^{\delta}$ be the universal curve, i.e., $\mathcal{C}$ is the subscheme

$$
\mathcal{C}=\{(p,[C]): p \in C\} \subset \mathbb{C P}^{2} \times \mathbb{C P}^{\delta}
$$

with a natural map to $\mathbb{C P}^{\delta}$. Here, $[C]$ denotes the polynomial defining the curve $C$. Thus the fiber of $\mathcal{C} \rightarrow \mathbb{C P}^{\delta}$ over $[C] \in \mathbb{C P}^{\delta}$ is the curve $C$. Let $\operatorname{Hilb}^{i}\left(\mathbb{C P}^{2}\right)$ be the Hilbert scheme of $i$ points in $\mathbb{C P}^{2}$. Finally, let $\operatorname{Hilb}^{i}\left(\mathcal{C} / \mathbb{C P}^{\delta}\right)$ be the relative Hilbert scheme

$$
\operatorname{Hilb}^{i}\left(\mathcal{C} / \mathbb{C P}^{\delta}\right)=\{(\mathbf{p},[C]): \mathbf{p} \subset C\} \subset \operatorname{Hilb}^{i}\left(\mathbb{C P}^{2}\right) \times \mathbb{C P}^{\delta},
$$

parametrizing curves in $\mathbb{C P}^{\delta}$ with $i$ marked points $\mathbf{p}$.

In their proof [KST11] of the Göttsche conjecture [Göt98, Conjecture 2.1], Kool, Shende, and Thomas showed, partially based on [PT10], that, for $d \geq \delta$, the Severi degree $N^{d, \delta}$ can be computed as a coefficient of the generating function of the Euler characteristic of the relative Hilbert schemes Hilb $\left(\mathcal{C} / \mathbb{C P}^{\delta}\right)$. Specifically, they show [KST11, Theorem 3.4] that, for fixed $d \geq \delta$, there exist integers $n_{r}$, for $r=g-\delta, \ldots, g$, with $g=g(d)=\left(\begin{array}{c}d-1 \\ 2\end{array}\right)$ the arithmetic genus of a degree- $d$ plane curve, and the following property:

$$
\sum_{i=0}^{\infty} e\left(\operatorname{Hilb}^{i}\left(\mathcal{C} / \mathbb{C P}^{\delta}\right)\right) z^{i}=\sum_{r=g-\delta}^{g} n_{r} z^{g-r}(1-z)^{2 r-2} .
$$

Here, $e(-)=\sum_{i \geq 0}(-1)^{i} \mathrm{rk} H^{i}(-, \mathbb{Z})$ denotes the topological Euler characteristic. Furthermore, they showed that the Severi degree $N^{d, \delta}$, for $d \geq \delta$, equals the coefficient $n_{g-\delta}$ in 2.1) (and that, in fact, a similar results holds for any smooth projective surface). 
Inspired by this description, Göttsche and Shende [GS11] suggest to replace in (2.1) the Euler characteristic $e(-)$ by

$$
\chi_{-y}(-)=\sum_{p, q \geq 0}(-1)^{p+q} y^{q} h^{p, q}(-),
$$

where $h^{p, q}(-)$ are the Hodge numbers. The polynomial $\chi_{-y}$ is the Hodge polynomial $H(\tilde{x}, \tilde{y})(-)=$ $\sum_{p, q \geq 0} \tilde{x}^{p} \tilde{y}^{q} h^{p, q}(-)$, at $\tilde{x}=-1$ and $\tilde{y}=-y$. They further suggest to define the following.

Definition 2.1 (Göttsche-Shende) For $d \geq 1$ and $\delta \geq 0$, let $\tilde{N}^{d, \delta}(y)$ be the polynomial $n_{g-\delta}(y)$ in $y$ in

$$
\sum_{i=0}^{\infty} \chi_{-y}\left(\operatorname{Hilb}^{i}\left(\mathcal{C} / \mathbb{C P}^{\delta}\right)\right) z^{i}=\sum_{r=g}^{g} n_{r}(y) z^{g-r}(1-z)^{r-1}(1-z y)^{r-1}
$$

where $n_{0}(y), \ldots, n_{g(d)}(y)$ are polynomials in $y$.

Following Göttsche and Shende, we call the polynomials $\tilde{N}^{d, \delta}(y)$ refined invariants (of $\mathbb{C P}^{2}$ ). Göttsche and Shende conjecture that $n_{r}(y)=0$ for $0 \leq r<g(d)-\delta$ as in 2.1. To emphasize the $q$-analogy, we from now on write $\tilde{N}^{d, \delta}(q)$ for $\tilde{N}^{d, \delta}(y)$. At $q=1$ we have $\chi_{-1}(-)=e(-)$ and thus recover the Severi degree as the special case $\tilde{N}^{d, \delta}(1)=N^{d, \delta}$, for $d \geq \delta$, from [KST11, Theorem 3.4]. The $\tilde{N}^{d, \delta}(q)$ automatically satisfy universal polynomiality [GS11]: for each $\delta$, there is a polynomial $\tilde{N}_{\delta}(d ; q)$ in $d$ and $y$ such that $\tilde{N}_{\delta}(d ; q)=\tilde{N}^{d, \delta}(q)$, provided that $d \geq \delta$.

Göttsche and Shende also conjecture a Caporaso-Harris type recursion for refined Severi degrees (Formula 2.2. We can use this recursion to define another family of polynomials $N^{d, \delta}(q)$, which Göttsche and Shende call refined Severi degrees. They conjecturally equal the refined invariants $\tilde{N}^{d, \delta}(q)$ (c.f. Conjecture 2.3. As in the non-refined case, the recursion involves relative refined Severi degrees $N^{d, \delta}(\alpha ; \beta)(q)$. These polynomials depend on two sequences $\alpha=\left(\alpha_{1}, \alpha_{2}, \ldots\right)$ and $\beta=\left(\beta_{1}, \beta_{2}, \ldots\right)$ of non-negative integers with finite support and $d=\sum_{i \geq 1} i\left(\alpha_{i}+\beta_{i}\right)$.

Before we state the formula, let us fix notation. For a sequence $\alpha=\left(\alpha_{1}, \alpha_{2}, \ldots\right)$, we write $I_{\alpha}=$ $1 \alpha_{1}+2 \alpha_{2}+\cdots$ as well as $|\alpha|=\alpha_{1}+\alpha_{2}+\cdots$. If $\alpha^{\prime}$ is another such sequence, then $\alpha^{\prime} \leq \alpha$ if and only if $\alpha_{i}^{\prime} \leq \alpha_{i}$ for all $i \geq 1$. For sequences $\alpha^{\prime} \leq \alpha$, let $\left(\begin{array}{c}\alpha \\ \alpha^{\prime}\end{array}\right)=\prod_{i \geq 1}\left(\begin{array}{c}\alpha_{i} \\ \alpha_{i}^{\prime}\end{array}\right)$. Let $e_{k}$ be the sequence $(0, \ldots, 0,1,0, \ldots)$ with 1 at position $k$. We usually omit writing down trailing zeros. For a positive integer $n$, we write $[n]_{q}=\frac{1-q^{n}}{1-q}$ for its $q$-number. Notice that $[n]_{1}=n$. Göttsche and Shende propose the following formula.

\section{Formula 2.2 (Göttsche-Shende-Caporaso-Harris Recursion)}

$$
\begin{aligned}
N^{d, \delta}(\alpha ; \beta)(q)= & \sum_{k: \beta_{k}>0}[k]_{q} \cdot N^{d, \delta}\left(\alpha+e_{k} ; \beta-e_{k}\right)(q) \\
& +\sum q^{I_{\alpha^{\prime}}+I_{\beta}} \prod_{i \geq 1}\left([i]_{q}\right)^{\beta_{i}} \cdot\left(\begin{array}{c}
\alpha \\
\alpha^{\prime}
\end{array}\right)\left(\begin{array}{c}
\beta^{\prime} \\
\beta
\end{array}\right) N^{d-1, \delta^{\prime}}\left(\alpha^{\prime} ; \beta^{\prime}\right)(q),
\end{aligned}
$$

the second sum over all sequences $\alpha^{\prime}, \beta^{\prime} \geq 0$ and integers $\delta^{\prime} \geq 0$ with

- $\alpha^{\prime} \leq \alpha, \beta^{\prime} \geq \beta$,

- $I_{\alpha^{\prime}}+I_{\beta^{\prime}}=d-1$,

- $\delta-\delta^{\prime}=d-1-\left|\beta-\beta^{\prime}\right|$,

together with the initial condition $N^{1,0}((1),(0))(q)=1$.

Formula 2.2 defines, for each $d \geq 1, \delta \geq 0$, and sequences $\alpha$ and $\beta$ of positive integers with $\sum_{i \geq 1} i\left(\alpha_{i}+\right.$ $\left.\beta_{i}\right)=d$, the relative refined Severi degree $N^{d, \delta}(\alpha ; \beta)(q)$, again a polynomial in $q$. Following the nonrefined case, we define $N^{d, \delta}(q)=N^{d, \delta}((0) ;(d))(q)$. Göttsche and Shende call the polynomials $N^{d, \delta}(q)$ refined Severi degrees. 
Conjecture 2.3 (Göttsche-Shende) For any $\delta \geq 0$ and $d \geq \frac{\delta}{2}+1$, we have

$$
N^{d, \delta}(q)=\tilde{N}^{d, \delta}(q)
$$

Conjecture 2.3 was verified by Göttsche and Shende for $d \leq 14$ and $\delta \leq 10$. Using a combinatorial description of the refined Severi degrees $N^{d, \delta}(q)$ defined by the Göttsche-Shende-Caporaso-Harris recursion (Formula 2.2), we extend this to $\delta \leq 4$ and all $d \geq \frac{\delta}{2}+1$ (c.f. Corollary 1.2 ).

Specializing Formula 2.2 at $q=1$ yields the famous classical recursion of Caporaso and Harris [CH98] for relative Severi degrees $N^{d, \delta}(\alpha ; \beta)$. These numbers count plane curves that, in addition to point condition, satisfying tangency conditions given by the "tangency profiles" $\alpha$ and $\beta$ to a fixed line $L$. Specifically, $\alpha_{i}$ resp. $\beta_{i}$ count the points where the curve is tangent to $L$ of order $i$ at fixed resp. unconstrained points (for details see [CH98]). A generic plane degree- $d$ curve intersects $L$ in $d$ simple nodes and thus $N^{d, \delta}((0) ;(d))=$ $N^{d, \delta}$.

\section{$3 q$-Weighted Floor Diagrams}

We now briefly review the marked floor diagrams of Brugallé and Mikhalkin [BM07, BM09]. We present them in the notation of Fomin and Mikhalkin [FM10]. A larger part of the definitions below is taken directly from [Blo11b].

Definition 3.1 A floor diagram $\mathcal{D}$ on a vertex set $\{1, \ldots, d\}$ is a directed graph (possibly with multiple edges) with positive integer edge weights wt $(e)$ satisfying:

1. The edge directions respect the order of the vertices, i.e., for each edge $i \rightarrow j$ of $\mathcal{D}$ we have $i<j$.

2. (Divergence Condition) For each vertex $j$ of $\mathcal{D}$, we have

$$
\operatorname{div}(j) \stackrel{\text { def }}{=} \sum_{\substack{\text { edges } e \\ j \stackrel{e}{\rightarrow} k}} \mathrm{wt}(e)-\sum_{\substack{\text { edges } e \\ i \stackrel{e}{\rightarrow} j}} \mathrm{wt}(e) \leq 1 .
$$

This means that at every vertex of $\mathcal{D}$ the total weight of the outgoing edges is larger by at most 1 than the total weight of the incoming edges.

The degree of a floor diagram $\mathcal{D}$ is the number of its vertices. It is connected if its underlying graph is. If $\mathcal{D}$ is connected its genus is the genus of the underlying graph. The cogenus of a connected floor diagram $\mathcal{D}$ of degree $d$ and genus $g$ is given by $\delta(\mathcal{D})=\frac{(d-1)(d-2)}{2}-g$. If $\mathcal{D}$ is not connected, let $d_{1}, d_{2}, \ldots$ and $\delta_{1}, \delta_{2}, \ldots$ be the degrees and cogenera, respectively, of its connected components. Then the cogenus of $\mathcal{D}$ is $\sum_{j} \delta_{j}+\sum_{j<j^{\prime}} d_{j} d_{j^{\prime}}$.

We now define our new statistic on $\mathcal{D}$ yielding a combinatorial formula for the refined Severi degrees $N^{d, \delta}(q)$. Recall that $[n]_{q}=\frac{1-q^{n}}{1-q}$.

Definition 3.2 We define the refined multiplicity $\mu(\mathcal{D}, q)$ of a floor diagram $\mathcal{D}$ as

$$
\mu(\mathcal{D}, q)=q^{\delta(\mathcal{D}))+\sum_{e}(1-\mathrm{wt}(e))} \cdot \prod_{\text {edges } e}\left([\operatorname{wt}(e)]_{q}\right)^{2}
$$

Notice that the weight $\mu(\mathcal{D}, q)$ is a polynomial in $q$ with positive integral coefficients. We draw floor diagrams using the convention that vertices in increasing order are arranged left to right. Edge weights of 1 are omitted.

Example 3.3 An example of a floor diagram of degree $d=4$, genus $g=1$, cogenus $\delta=2$, divergences $1,1,0,-2$, and multiplicity $\mu(\mathcal{D} ; q)=(1+q)^{2}$ is drawn below.

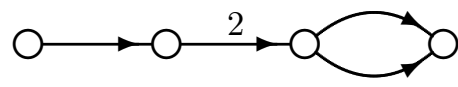


To a floor diagram, we associate a last statistic, the "number of its markings."

Definition 3.4 A marking of a floor diagram $\mathcal{D}$ is defined by the following three step process which we illustrate in the case of Example 3.3

Step 1: For each vertex $j$ of $\mathcal{D}$ create 1 - div $(j)$ many new vertices and connect them to $j$ with new edges directed away from $j$.

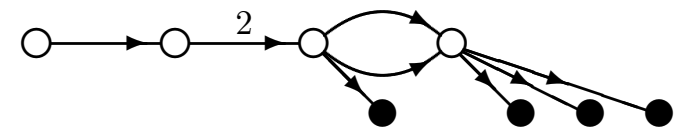

Step 2: Subdivide each edge of the original floor diagram $\mathcal{D}$ into two directed edges by introducing a new vertex for each edge. The new edges inherit their weights and orientations. Call the resulting graph $\widetilde{\mathcal{D}}$.

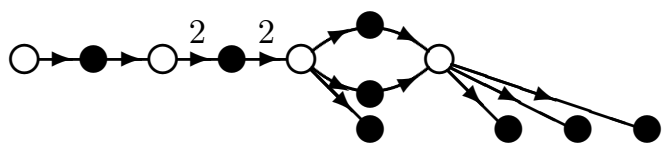

Step 3: Linearly order the vertices of $\widetilde{\mathcal{D}}$ extending the order of the vertices of the original floor diagram $\mathcal{D}$ such that, as before, each edge is directed from a smaller vertex to a larger vertex.

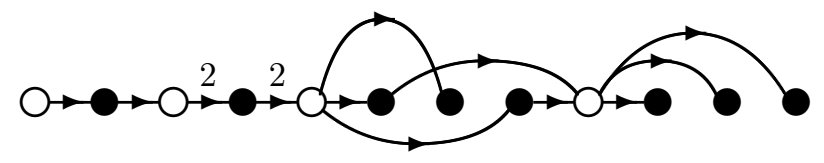

The extended graph $\widetilde{\mathcal{D}}$ together with the linear order on its vertices is called a marked floor diagram, or $a$ marking of the original floor diagram $\mathcal{D}$.

We want to count marked floor diagrams up to equivalence. Two markings $\widetilde{\mathcal{D}}_{1}, \widetilde{\mathcal{D}}_{2}$ of a floor diagram $\mathcal{D}$ are equivalent if there exists an automorphism of weighted graphs which preserves the vertices of $\mathcal{D}$ and maps $\widetilde{\mathcal{D}}_{1}$ to $\widetilde{\mathcal{D}}_{2}$. The number of markings $\nu(\mathcal{D})$ is the number of marked floor diagrams $\widetilde{\mathcal{D}}$ up to equivalence.

Example 3.5 The floor diagram $\mathcal{D}$ of Example 3.3 has $\nu(\mathcal{D})=7$ markings (up to equivalence): In step 3 the extra 1-valent vertex connected to the third white vertex from the left can be inserted in three ways between the third and fourth white vertex (up to equivalence) and in four ways right of the fourth white vertex (again up to equivalence).

Denote by $\mathbf{F D}(d, \delta)$ the set of floor diagrams $\mathcal{D}$ of degree $d(\mathcal{D})=d$ with cogenus $\delta(\mathcal{D})=\delta$. The statistic $\nu(\mathcal{D})$ on $\mathbf{F D}(d, \delta)$ can be generalized to the number $\nu_{\alpha, \beta}(\mathcal{D})$ of " $(\alpha, \beta)$-markings" of $\mathcal{D}$, for any two non-negative integral sequences $\alpha$ and $\beta$ with $\sum_{i \geq 1} i\left(\alpha_{i}+\beta_{i}\right)=d$. Basically, one allows, in Step 1 of Definition 3.4, new edges with weight bigger than 1. The precise definition can be found in [Blo12, Definition 2.3]. Not surprisingly, such markings count plane curves satisfying tangency conditions to a fixed line [Blo12, Theorem 2.4].

Now we are ready for the most important definition of the paper, a combinatorial notion of refined Severi degrees. Later, we will see that this notion coincides with $N^{d, \delta}(q)$ and, thus, conjecturally with the refined invariants $\tilde{N}^{d, \delta}(q)$ for $d \geq \frac{\delta}{2}+1$ of Göttsche and Shende.

Definition 3.6 For $d \geq 1$ and $\delta \geq 0$, we define the combinatorial refined Severi degree $N_{\mathrm{comb}}^{d, \delta}(q)$ to be the polynomial in q given by

$$
N_{\mathrm{comb}}^{d, \delta}(q)=\sum_{\mathcal{D} \in \mathbf{F D}(d, \delta)} \mu(\mathcal{D} ; q) \cdot \nu(\mathcal{D}) .
$$

Furthermore, for non-negative sequences $\alpha$ and $\beta$ with finite support, we define the relative combinatorial refined Severi degree $N_{\text {comb }}^{d, \delta}(\alpha ; \beta)(q)$ to be the polynomial in $q$ given by

$$
N_{\mathrm{comb}}^{d, \delta}(\alpha ; \beta)(q)=\prod_{i \geq 1}\left([i]_{q}\right)^{\beta_{i}} \sum_{\mathcal{D} \in \mathbf{F D}(d, \delta)} \mu(\mathcal{D} ; q) \cdot \nu_{\alpha, \beta}(\mathcal{D}) .
$$


It is straightforward to check that in the non-relative case, where $\alpha=(0,0, \ldots)$ and $\beta=(d, 0,0, \ldots)$, (3.1) and 3.2 agree.

Observation 3.7 Both (3.1) and (3.2) are positive combinatorial formulas. Even more is true: it is clear from the definition that $N_{\mathrm{comb}}^{d, \delta}(q)$ and $N_{\mathrm{comb}}^{d, \delta}(\alpha ; \beta)(q)$ are polynomials in $q$ with positive integral coefficients. Of course, a natural question is: what do the coefficients count?

The following is our key result.

Theorem 3.8 The polynomials $N_{\text {comb }}^{d, \delta}(\alpha ; \beta)(q)$ satisfy Formula 2.2 Therefore, we have $N_{\text {comb }}^{d, \delta}(\alpha ; \beta)(q)=$ $N^{d, \delta}(\alpha ; \beta)(q)$.

Proof: This follows from a lengthy, quite straightforward computation. Details will appear in the forthcoming paper [BG12].

In the sequel, we will usually write $\tilde{N}$ instead of $N_{\text {comb }}$ even while referring to the combinatorial defined refined Severi degree if no confusion can occur.

It is easy to see that, at $q=1$ resp. $q=-1$, the refined multiplicity $\mu(\mathcal{D}, q)$ times the number of markings $\nu(\mathcal{D})$ of $\mathcal{D}$ specializes to the "complex" resp. "real multiplicity" of Brugallé and Mikhalkin [BM09, Definitions 3.5 and 3.8]. From their combinatorial description of the (non-relative) Severi degree $N^{d, \delta}$ in terms of floor diagrams, we immediately conclude the following.

Theorem 3.9 ([BM09, Theorem 3.6]) We have $N_{\text {comb }}^{d, \delta}(1)=N^{d, \delta}$.

An alternate way to see this is to notice that the refined recursion (Formula 2.2 defining $N^{d, \delta}(q)$ specializes, at $q=1$, to the classical Caporaso-Harris recursion [CH98, Theorem 1.1], which computes the Severi degrees $N^{d, \delta}$.

Before turning to the polynomiality in $d$ of the refined Severi degrees $N^{d, \delta}(q)$ in the next section, we briefly discuss the specialization $q=0$. Göttsche and Shende [GS11] proved that the refined invariant $\tilde{N}^{d, \delta}(0)$ equals $\left(\begin{array}{c}g(d) \\ \delta\end{array}\right)$, where $g(d)=\left(\begin{array}{c}d-1 \\ 2\end{array}\right)$ is the geometric genus of a plane curve of degree $d$. (They, in fact, gave a formula for all smooth projective surfaces.) They further showed that the refined Severi degree $N^{d, \delta}(0)$ is given by the same formula. Using our combinatorial description of the refined Severi degrees, we can give an alternate proof of the latter result. Yet another proof can be obtained directly via the refined Caporaso-Harris recursion at $q=0$.

Proposition 3.10 (Göttsche-Shende [GS11]) We have $N^{d, \delta}(0)=\left(\begin{array}{c}g(d) \\ \delta\end{array}\right)$, with $g(d)=\left(\begin{array}{c}d-1 \\ 2\end{array}\right)$.

Proof: See the forthcoming paper [BG12].

\section{Refined Node Polynomials for Plane Curves}

We now use the combinatorial formula 3.1 for the refined Severi degrees $N^{d, \delta}(q)$ to show that, for fixed $\delta$, they are given by polynomials $N_{\delta}(d ; q)$ in $d$ and $q$ (Theorem 1.1 . We call the polynomials $N_{\delta}(d ; q)$ "refined node polynomials". At $q=1$, they specializes to the usual node polynomials $N_{\delta}(d)$ of the introduction. Our technique is a quite straightforward $q$-analog extension of Fomin and Mikhalkin's method [FM10, Section 5]. In particular, it provides an algorithm to compute refined node polynomials for any $\delta$; see Remark 4.5 for a list for $\delta \leq 2$. For begin by reviewing Fomin and Mikhalkin's key gadget.

Definition $4.1 A$ template $\Gamma$ is a directed graph (possibly with multiple edges) on vertices $\{0, \ldots, l\}$, where $l \geq 1$, with edge weights wt $(e) \in \mathbb{Z}_{>0}$, satisfying:

1. If $i \rightarrow j$ is an edge, then $i<j$.

2. Every edge $i \stackrel{e}{\rightarrow} i+1$ has weight $\mathrm{wt}(e) \geq 2$. (No "short edges.")

3. For each vertex $j, 1 \leq j \leq l-1$, there is an edge "covering" it, i.e., there exists an edge $i \rightarrow k$ with $i<j<k$. 
Every template $\Gamma$ comes with some numerical data associated with it. Its length $l(\Gamma)$ is the number of vertices minus 1 . Its cogenus $\delta(\Gamma)$ is

$$
\delta(\Gamma)=\sum_{i \rightarrow j}[(j-i) \mathrm{wt}(e)-1] .
$$

Notice that, for each $\delta$, there are only a finite number of templates with cogenus $\delta$. We define its q-multiplicity $\mu(\Gamma, q)$ to be

$$
\mu(\Gamma, q)=q^{\delta(\Gamma)}\left[\prod_{\text {edges } e}\left([\operatorname{wt}(e)]_{q}\right)^{2} \cdot q^{1-\mathrm{wt}(e)}\right]
$$

At $q=1$, we recover Fomin and Mikhalkin's template multiplicity $\prod_{e} \mathrm{wt}(e)^{2}$. It follows from 4.1) that, for every template $\Gamma, \mu(\Gamma, q)$ is a polynomial with positive integral coefficients.

For $1 \leq j \leq l(\Gamma)$, let $\varkappa_{j}=\varkappa_{j}(\Gamma)$ denote the sum of the weights of edges $i \rightarrow k$ with $i<j \leq k$. So $\varkappa_{j}(\Gamma)$ equals the total weight of the edges of $\Gamma$ from a vertex left of $j$ to a vertex right of or equal to $j$. Define

$$
k_{\min }(\Gamma)=\max _{1 \leq j \leq l}\left(\varkappa_{j}-j+1\right) .
$$

This makes $k_{\min }(\Gamma)$ the smallest positive integer $k$ such that $\Gamma$ can appear in a floor diagram on $\{1,2, \ldots\}$ with left-most vertex $k$. Lastly, set

$$
\varepsilon(\Gamma)= \begin{cases}1 & \text { if all edges arriving at } l \text { have weight } 1 \\ 0 & \text { otherwise }\end{cases}
$$

See [FM10, Figure 10] for a list of all templates $\Gamma$ with $\delta(\Gamma) \leq 2$.

A labeled floor diagram $\mathcal{D}$ with $d$ vertices decomposes into an ordered collection $\left(\Gamma_{1}, \ldots, \Gamma_{m}\right)$ of templates as follows: First, add an additional vertex $d+1(>d)$ to $\mathcal{D}$ along with, for every vertex $j$ of $\mathcal{D}$, $1-\operatorname{div}(j)$ new edges of weight 1 from $j$ to the new vertex $d+1$. The resulting floor diagram $\mathcal{D}^{\prime}$ has divergence 1 at every vertex coming from $\mathcal{D}$. Now remove all short edges from $\mathcal{D}^{\prime}$, that is, all edges of weight 1 between consecutive vertices. The result is an ordered collection of templates $\left(\Gamma_{1}, \ldots, \Gamma_{m}\right)$, listed left to right, and it is not hard to see that $\sum \delta\left(\Gamma_{i}\right)=\delta(\mathcal{D})$. This process is reversible once we record the smallest vertex $k_{i}$ of each template $\Gamma_{i}$ (see Example 4.2.

Example 4.2 An example of the decomposition of a labeled floor diagram into templates is illustrated below. Here, $k_{1}=2$ and $k_{2}=4$.

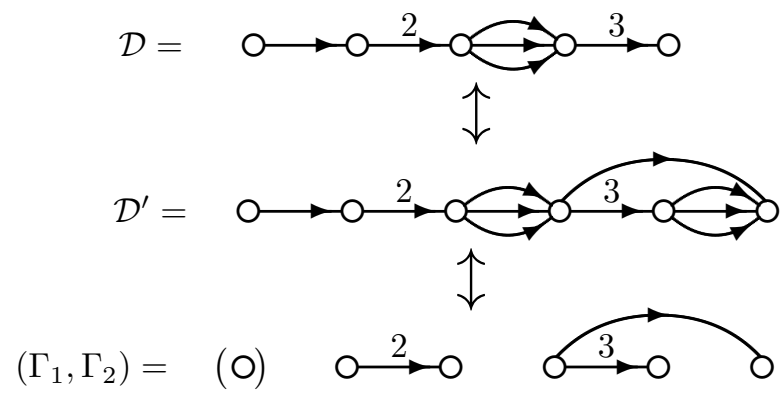

To each template $\Gamma$ we associate a polynomial that records the number of "markings of $\Gamma$ :" For $k \in \mathbb{Z}_{>0}$ let $\Gamma^{(k)}$ denote the graph obtained from $\Gamma$ by first adding $k+i-1-\varkappa_{i}$ short edges connecting $i-1$ to i, for $1 \leq i \leq l(\Gamma)$, and then subdividing each edge of the resulting graph by introducing one new vertex for each edge. By [FM10, Lemma 5.6] the number of linear extensions (up to equivalence) of the vertex poset of the graph $\Gamma^{(k)}$ extending the vertex order of $\Gamma$ is a polynomial in $k$, if $k \geq k_{\min }(\Gamma)$, which we denote by $p(\Gamma, k)$ (see [FM10, Figure 10] for examples). 
The invariants of a floor diagram $\mathcal{D}$ are compatible with the invariants of its template decomposition $\left\{\left(\Gamma_{i}, k_{i}\right)\right\}_{i=1}^{m}$, in the following sense. We have $\delta(\mathcal{D})=\sum_{i=1}^{m} \delta\left(\Gamma_{i}\right)[$ FM10, Lemma 5.7]. Furthermore, as each marking of $\mathcal{D}$ can be obtained by ordering the vertices of each $\Gamma_{i}^{\left(k_{i}\right)}$ individually, we have

$$
\nu(\mathcal{D})=\prod_{i=1}^{m} p\left(\Gamma_{i}, k_{i}\right)
$$

Finally, the proof of the following lemma is straightforward.

Lemma 4.3 With the notation from above, we have

$$
\mu(\mathcal{D}, q)=\prod_{i=1}^{m} \mu\left(\Gamma_{i}, q\right)
$$

We record, for each ordered template collection $\boldsymbol{\Gamma}=\left(\Gamma_{1}, \ldots, \Gamma_{m}\right)$, all valid "positions" $k_{i}$ that can occur in the template decomposition of a floor diagram of degree $d$ by the lattice points in the polytope

$$
\begin{aligned}
P_{\boldsymbol{\Gamma}}(d)=\left\{\mathbf{k} \in \mathbb{R}^{m}:\right. & k_{i} \geq k_{\min }\left(\Gamma_{i}\right), \\
& \left.k_{i}+l\left(\Gamma_{i}\right) \leq k_{i+1}(1 \leq i<m), k_{m}+l\left(\Gamma_{m}\right) \leq d+\varepsilon\left(\Gamma_{m}\right)\right\} .
\end{aligned}
$$

The first inequality says that, due to the divergence condition, templates cannot appear to early in a floor diagram. The second resp. third inequality say that templates cannot overlap resp. cannot hang over at the end of the floor diagram. The following proposition follows from Definition 3.6. Theorem 3.8, Lemma 4.3 and the previous discussion.

Proposition 4.4 For any $d \geq 1$ and $\delta \geq 1$, the refined Severi degree $N^{d, \delta}(q)$ is given by

$$
N^{d, \delta}(q)=\sum_{\Gamma: \sum_{i} \delta\left(\Gamma_{i}\right)=\delta}\left[\left(\prod_{i=1}^{m} \mu\left(\Gamma_{i}, q\right)\right) \sum_{\mathbf{k} \in P_{\Gamma}(d) \cap \mathbb{Z}^{m}}\left(\prod_{i=1}^{m} p\left(\Gamma_{i}, k_{i}\right)\right)\right] .
$$

Here, the first sum is over all templates collections $\boldsymbol{\Gamma}=\left(\Gamma_{1}, \ldots, \Gamma_{m}\right)$ with $\sum_{i=1}^{m} \delta\left(\Gamma_{i}\right)=\delta$.

For $q=1$, expression (4.2) specializes to [FM10, (5.13)]. In fact, our argument above closely follows that of [FM10]. The proof of Theorem 1.1 is now easy.

Proof Proof of 1.1 (Sketch): For fixed $\delta$, the only non-finite expression in 4.2 is the second sum. This sum is an iterated discrete integral of the polynomial $\prod_{i} p\left(\Gamma_{i}, k_{i}\right)$ over the unimodular simplex $P_{\Gamma}(d)$. (A discrete integral is a function of the form $f(d)=\sum_{k=k_{0}}^{d} g(k)$ for some function $g$.) The result now follows, for example, by Faulhaber's formula ([Blo11b, Lemma 3.5], taken from [Knu93]), which implies that discrete integration preserves polynomiality for large $d$. The precise threshold follows by a similar argument as in [Blo11b, Section 4].

Remark 4.5 Expression (4.2) gives, in principle, an algorithm to compute the relative node polynomials $\tilde{N}_{\delta}(d ; q)$. The algorithm of [Blo11b] Section 3], based on the algorithm of Fomin and Mikhalkin [FM10] Section 5], easily adapts to the $q$-analog case. Below we show $\tilde{N}_{\delta}(d ; q)$ for $\delta \leq 2$ (the author computed for $\delta \leq 7$, see [Blolla]):

$$
\begin{aligned}
\tilde{N}_{1}(d ; q)= & \frac{1}{2} q^{2} d^{2}-\frac{3}{2} q^{2} d+q^{2}+2 q d^{2}-3 q d+q+\frac{1}{2} d^{2}-\frac{3}{2} d+1, \\
\tilde{N}_{2}(d ; q)= & \frac{1}{8} q^{4} d^{4}-\frac{3}{4} q^{4} d^{3}+\frac{11}{8} q^{4} d^{2}-\frac{3}{4} q^{4} d+q^{3} d^{4}-\frac{9}{2} q^{3} d^{3}+2 q^{3} d^{2}+\frac{21}{2} q^{3} d-9 q^{3}+\frac{9}{4} q^{2} d^{4} \\
& -\frac{15}{2} q^{2} d^{3}-\frac{3}{4} q^{2} d^{2}+21 q^{2} d-15 q^{2}+q d^{4}-\frac{9}{2} q d^{3}+2 q d^{2}+\frac{21}{2} q d-9 q+\frac{1}{8} d^{4}-\frac{3}{4} d^{3} \\
& +\frac{11}{8} d^{2}-\frac{3}{4} d .
\end{aligned}
$$


Remark 4.6 We expect our methods to generalize to toric surfaces of " $h$-transverse" polygons, in the sense of [BM09]. Such surfaces are in general not smooth and include $\mathbb{C P}^{1} \times \mathbb{C P}^{1}$ and Hirzebruch surfaces. Specifically, we expect that, by a q-analog of the methods of [AB10], one can construct combinatorially defined polynomials that, conjecturally, compute the "refined Severi degree of such surfaces." In the smooth case, this should agree, for sufficiently ample line bundles, with Göttsche and Shende's definition of refined Severi degrees via replacing, in [KST11. Theorem 3.4], the topological Euler characteristic by the Hodge polynomial (2.2).

\section{Acknowledgements}

The author is grateful to Lothar Göttsche, for explaining his work and for providing the slides of his talks. He thanks Ilia Itenberg, Martijn Kool, and Damiano Testa for helpful discussions, the anonymous referee for helpful suggestions, and Diane Maclagan for telling the author about this problem.

\section{References}

[AB10] F. Ardila and F. Block. Universal polynomials for Severi degrees of toric surfaces. Preprint, arXiv:1012.5305, 2010.

[BG12] F. Block and L. Göttsche. Refined Severi degrees for plane curves. In preparation, 2012.

[Blo11a] F. Block, 2011. http://www.warwick.ac.uk/staff/F.S.Block/RefinedNP.zip.

[Blo11b] F. Block. Computing node polynomials for plane curves. Math. Res. Lett., 18:621-643, 2011.

[Blo12] F. Block. Relative node polynomials for plane curves. J. Algebraic Combin. (to appear), 2012.

[BM07] E. Brugallé and G. Mikhalkin. Enumeration of curves via floor diagrams. C. R. Math. Acad. Sci. Paris, 345(6):329-334, 2007.

[BM09] E. Brugallé and G. Mikhalkin. Floor decompositions of tropical curves: the planar case. In Proceedings of Gökova Geometry-Topology Conference 2008, pages 64-90. Gökova Geometry/Topology Conference (GGT), Gökova, 2009.

[CH98] L. Caporaso and J. Harris. Counting plane curves of any genus. Invent. Math., 131(2):345-392, 1998.

[DFI95] P. Di Francesco and C. Itzykson. Quantum intersection rings. In The moduli space of curves (Texel Island, 1994), volume 129 of Progr. Math., pages 81-148. Birkhäuser Boston, Boston, MA, 1995.

[FM10] S. Fomin and G. Mikhalkin. Labeled floor diagrams for plane curves. J. Eur. Math. Soc. (JEMS), 12(6):1453-1496, 2010.

[Göt98] L. Göttsche. A conjectural generating function for numbers of curves on surfaces. Comm. Math. Phys., 196(3):523-533, 1998.

[GS11] L. Göttsche and V. Shende. Work in progress. 2011.

[IKS09] I. Itenberg, V. Kharlamov, and E. Shustin. A Caporaso-Harris type formula for Welschinger invariants of real toric del Pezzo surfaces. Comment. Math. Helv., 84(1):87-126, 2009.

[Knu93] D. Knuth. Johann Faulhaber and sums of powers. Math. Comp., 61(203):277-294, 1993.

[KST11] M. Kool, V. Shende, and R. P. Thomas. A short proof of the Göttsche conjecture. Geom. Topol., 15:397-406, 2011.

[PT10] R. Pandharipande and R. P. Thomas. Stable pairs and BPS invariants. J. Amer. Math. Soc., 23(1):267-297, 2010. 
[Shu05] E. Shustin. A tropical approach to enumerative geometry. Algebra i Analiz, 17(2):170-214, 2005.

[Ste48] J. Steiner. Elementare Lösung einer geometrischen Aufgabe, und über einige damit in Beziehung stehende Eigenschaften der Kegelschnitte. J. Reine Angew. Math., 37:161-192, 1848.

[Tze10] Y.-J. Tzeng. A proof of Göttsche-Yau-Zaslow formula. Preprint, arXiv:1009.5371, 2010. 
